\title{
Essais
}

Revue interdisciplinaire d'Humanités

Hors-série 6 | 2021

Agrobiodiversité et territoires

\section{L'usage de savoirs paysans dans la réflexion sur le devenir des races anciennes de poules en France}

D'un constat de déclin à une réflexion sur les éléments d'une nouvelle économie et d'une nouvelle organisation spatiale de l'élevage s'appuyant sur la complémentarité de territoires

The use of farmers' knowledge in the reflection on the future of older breeds of chickens in France

From an observation of decline to a reflection on the elements of a new economy and a new spatial organisation of livestock farming based on the complementarity of territories

Dominique Dubuc et Hervé Goulaze

\section{OpenEdition}

Journals

Édition électronique

URL : https://journals.openedition.org/essais/7573

DOI : 10.4000 /essais. 7573

ISSN : 2276-0970

Éditeur

École doctorale Montaigne Humanités

Édition imprimée

Date de publication : 1 mars 2021

Pagination : 111-123

ISBN : 978-2-492780-00-4

ISSN : 2417-4211

Référence électronique

Dominique Dubuc et Hervé Goulaze, "L'usage de savoirs paysans dans la réflexion sur le devenir des races anciennes de poules en France », Essais [En ligne], Hors-série 6 | 2021, mis en ligne le 16 mars 2021, consulté le 18 janvier 2023. URL : http://journals.openedition.org/essais/7573 ; DOI : https:// doi.org/10.4000/essais.7573 


\title{
L'usage de savoirs paysans dans la réflexion sur le devenir des races anciennes de poules en France
}

\author{
$D^{\prime}$ un constat de déclin à une réflexion sur les éléments d'une \\ nouvelle économie et d'une nouvelle organisation spatiale de \\ l'élevage s'appuyant sur la complémentarité de territoires
}

\section{Dominique Dubuc, Hervé Goulaze}

Les savoirs paysans permettent d'enrichir la réflexion s'intéressant à l'agrobiodiversité dans les territoires d'une façon complémentaire de la connaissance scientifique. Guy Kastler, un paysan engagé, expose que les savoirs paysans procèdent d'une connaissance issue des choix du paysan face au contexte/à l'environnement dans lequel il évolue. Il ne s'agit pas d'une connaissance scientifique mais d'un "savoir être et un savoir se comporter dans un écosystème dont le paysan lui-même fait partie " ${ }^{1}$. Les savoirs paysans " tiennent compte de multiples aléas et intègrent ces aléas à l'action quotidienne à long terme $»^{2}$. Un trait spécifique du savoir paysan tient à ce que son générateur, le paysan, n’est pas seulement en situation dans un contexte, dans un environnement, il est aussi un élément du contexte dans lequel il évolue et avec lequel il interagit ${ }^{3}$. Le savoir paysan est ainsi à la fois une connaissance qui provient de la confrontation au terrain et une connaissance qui façonne le terrain, qui influe sur son évolution. Estelle Deléage fait de ce savoir la raison de la meilleure adaptation de l'agriculture paysanne par rapport à "l'agriculture manufacturière " pour répondre aux problèmes actuels posés par la production alimentaire ${ }^{4}$.

Notre démarche utilise l'apport de savoirs paysans spécifiques à un pan de l'agrobiodiversité - l'élevage des races anciennes de poules - pour enrichir une réflexion sur une nouvelle intégration de cet élevage aux territoires, en particulier les territoires urbains et périurbains. En quoi l'apport de savoirs paysans peut aider à adapter les races anciennes de volailles françaises à un contexte spatial, économique, socio-culturel et paysager devenu très différent des contextes dans lesquels elles ont été créées ? Pourquoi l'usage de savoirs

1 Guy Kastler, Aliénor Bertrand, Sarah Vanuxem, "Des savoirs paysans ", in Cahiers philosophiques, 2018/1, n 152, "Le vegetal, savoirs et pratiques ", Vrin, p. 38-39.

2 Michèle Salmona, socio-anthropologue, citée par Estelle Deléage, « Les paysans dans la modernité ", in Revue française de socio-économie, 2012/1, n 9, p. 131.

3 Kastler, Bertrand et Vanuxem, op. cit.

4 Deléage, op. cit. 
paysans se montre pertinent au sein d'une réflexion sur la reconstitution d'un élevage attaché à maintenir un patrimoine et une diversité génétiques et gustatifs ? Nous regardons ce patrimoine, cette diversité comme faisant partie des fondements nécessaires à la naissance de nouveaux terroirs de production capables de proposer des productions saines et diversifiées dans leurs caractéristiques gustatives et nutritionnelles en particulier.

L'expérience d'un éleveur chevronné, Dominique Dubuc, est le support de notre démarche. Il porte sur l'élevage des races anciennes de volailles en France un regard fondé sur une longue expérience de terrain de soixantequinze ans. Chez ce paysan octogénaire le goût et la pratique de l'élevage remontent à son enfance. En 1997 il a fondé avec son épouse Geneviève le Conservatoire avicole du Puyobrau à Magescq (Landes) à partir de l'observation que les races anciennes de volailles se raréfiaient, notamment les races françaises. Il diffuse depuis lors de nombreux spécimens de ces races en France et en Europe occidentale.

Nous ne questionnons pas dans cet article le bien-fondé des savoirs paysans de Dominique Dubuc. Nous les considérons validés par la reconnaissance qu'il a obtenu auprès de ses pairs ainsi qu'auprès d'instances associatives reconnues pour la pertinence et la rigueur de leur travail de préservation de l'agrobiodiversité animale, en premier lieu le Conservatoire des Races d'Aquitaine dont Dominique Dubuc est membre. La réputation avantageuse du Conservatoire avicole du Puyobrau en matière de conservation et de diffusion des races de volailles repose sur un travail rigoureux de sélection et de reproduction, perpétuant des méthodes employées avant l'industrialisation de l'élevage avicole.

Notre approche se divise en deux volets. D'une part l'établissement par Dominique Dubuc d'un constat sur l'état de l'élevage des races françaises de poules aujourd'hui. Il est fondé sur sa pratique d'éleveur, ses observations, les informations qu'il a collectées dans le cadre de son activité professionnelle. Pour subjective qu'elle soit, cette analyse préalable repose sur cette perception et cette expérience venue de l'intérieur - forgée ici au cœur d'une activité d'élevage conservatoire de longue durée - qui constitue la matrice des savoirs paysans comme l'expliquent Guy Kastler et Estelle Deléage. Si les observations de Dominique Dubuc sur la situation des races anciennes de poules françaises rejoignent sur le fond les constats dressés par les institutions qui étudient cette question, sa connaissance des effectifs de nombreuses races et de la situation de leur élevage, fondée sur l'expérience de terrain, peut conduire à des différences d'appréciation significatives entre ces deux sources ${ }^{5}$.

Le second volet présente l'ébauche de plusieurs modèles d'élevage conçus pour intégrer un ensemble de contraintes actuelles qui affectent particulièrement les territoires urbains et périurbains français. Dominique Dubuc identi-

5 Cf. p. 3, 11 et 12, INRA. Races animales françaises menacées d'abandon: liste des races menacées d'abandon pour l'agriculture. INRA Science et impact, 2014. 
fie ces contraintes comme des exigences dont une prise en compte pertinente concourt de façon déterminante aux chances de succès des formes d'élevage envisagées.

\section{L'élevage des races françaises de poules : un panorama dessiné par Dominique Dubuc à partir de ses savoirs paysans}

Les races françaises de poules avant la massification de l'élevage des volailles

Toutes les races de poules françaises étaient utilitaires. Deux ensembles se distinguent parmi les races françaises anciennes, toutes créées avant la Seconde Guerre mondiale :

- les races coureuses, de ferme. Il s'agit de races rustiques qui cherchent elles-mêmes leur nourriture. La poule Landaise ou la poule de Caussade en sont deux exemples ;

- les races moins " débrouillardes ${ }^{6}$ ", issues de régions disposant de céréales en quantité, qui avaient été souvent sélectionnées pour approvisionner en volailles goûteuses les marchés des grandes villes. Les races de poules normandes ou d'Ile-de-France, la poule de Barbezieux en font partie.

En 1945 il existait 44 races de poules françaises qui pouvaient être qualifiées de races anciennes. La diversité de ces races correspondait à la diversité des terroirs où elles avaient été sélectionnées. Leur répartition était très inégale sur le territoire national : une abondance de races se rencontrait au Nord de la France tandis qu'elles étaient rares sur le pourtour méditerranéen.

À côté des races anciennes, sont également créées dans la seconde moitié $\mathrm{du} \mathrm{XX}^{\mathrm{e}}$ siècle quelques races locales à l'intérêt patrimonial limité, "nées de la fantaisie d'éleveurs chevronnés" " et non d'une longue sélection au sein de régions où les populations ainsi obtenues constituent une assise solide pour que naisse et se perpétue un terroir de production avicole. Ce sont les races Aquitaine, Charollaise, Cou-nu du Forez et Meusienne.

6 Le qualificatif est de Dominique Dubuc qui a établi lui-même cette distinction entre races françaises, fondée sur leur rusticité et les conditions d'élevage qui en résultent.

7 Alain Raveneau, Inventaire des animaux domestiques en France, Nathan, 2004 (2 $2^{\mathrm{e}}$ ed.), p. 267. 
Un constat : la disparition de la majeure partie des races françaises de poules

\begin{tabular}{|ll|}
\hline \multicolumn{2}{|l}{ Races françaises anciennes de poules encore représentées aujourd'hui } \\
Alsacienne & Gournay \\
Barbezieux & Hergnies \\
Bourbonnaise & Houdan \\
Bourbourg & Janzé \\
Bresse blanche à crete pale & La-Flèche \\
Bresse Gauloise (anc. Bresse) & Landaise \\
Caumont & Le-Mans \\
Caussade & Limousine \\
Caux & Lyonnaise \\
Contres & Mantes \\
Cotentine & Merlerault \\
Coucou des Flandres & Noire du Berry \\
Coucou de France & Pavilly \\
Coucou de Rennes & Pictave \\
Crèvecoeur & \\
Estaires & \\
Faverolles & \\
Gasconne & \\
Gatinaise & \\
Gauloise dorée & \\
\hline
\end{tabular}

Figure 1 : Races françaises anciennes de poules encore représentées aujourd'hui

\begin{tabular}{|lll}
\hline Races françaises de poules menacées ou non menacées à court terme \\
Races menacées & & Races non menacées \\
Aquitaine & Géline de Touraine & Alsacienne \\
Bresse blanche à crete pale & Hergnies & Barbezieux \\
Bourbonnaise & Houdan & Bresse gauloise \\
Bourbourg & Janzé & Coucou de Rennes \\
Caumont & La-Flèche & Gasconne \\
Caussade & Landaise & Gauloise dorée \\
Caux & Le-Mans & Gournay \\
Challans & Lyonnaise & Limousine \\
Charollaise & Mantes & Marans \\
Contres & Merlerault & Noire du Berry \\
Cotentine & Meusienne & \\
Coucou des Flandres & Pavilly & \\
Coucou de France & & \\
Cou-nu du Forez & & \\
Crèvecoeur & & \\
Estaires & & \\
Faverolles & & \\
Gatinaise & & \\
\hline
\end{tabular}

Figure 2 : Races françaises de poules menacées ou non menacées à court terme

Aujourd'hui 30 races françaises de poules sont menacées de disparition à court terme ; 10 seulement ne le sont pas. Si autant de races sont en grand danger de disparaître, cela tient à ce que l'on n'a pas réussi à leur faire occuper à nouveau un créneau économique qui assure une rentabilité à leur élevage. 
L'industrialisation de l'élevage avicole et son incidence néfaste sur la diversité et les effectifs des races anciennes de poules en France

La première raison tient à la massification de la production de viande de volailles et d'œufs sous l'effet de son industrialisation qui a porté un coup terrible aux races anciennes. Le poulet était, il y a soixante-cing ans encore, une des viandes les plus chères à l'achat. Du fait de son industrialisation elle compte aujourd'hui parmi les moins onéreuses. Cela signe la perte de qualité des produits de cet élevage.

La production de masse repose sur l'usage de souches industrielles à croissance rapide. Pour produire de la viande bon marché, on a réduit le temps d'élevage des sujets. La sélection de souches à croissance toujours plus rapide a tué les races anciennes.

Aujourd'hui, dans l'élevage industriel, un poulet peut être prêt à abattre à 27 jours alors qu'il faut 5 mois pour obtenir un poulet à partir d'une race ancienne. Les races françaises n'avaient aucune chance de concurrencer les souches industrielles sur le critère de la rapidité d'obtention des produits. L'aviculture est une des productions qui a le plus évolué dans l'agroalimentaire, au détriment de la qualité gustative et nutritionnelle des produits. La deuxième raison tient à ce que le renouvellement des éleveurs ne s'opère plus. Les anciens peuvent de moins en moins transmettre leur élevage à des jeunes. Les causes en sont multiples.

Un désintérêt pour l'élevage des races anciennes est de plus en plus manifeste, en particulier auprès des jeunes générations. L'élevage est un assujettissement : il faut s'occuper chaque jour des volailles alors que les conditions de vie actuelles et les déplacements quotidiens qu'elles imposent sont peu compatibles avec cette nécessité. Ce désintérêt toujours plus répandu amène à ce que des élevages toujours plus nombreux ne soient plus repris et disparaissent. Parmi les contraintes apportées par les conditions de vie actuelles la première en nuisance pour la pérennité des races anciennes reste la difficulté d'élever des coqs à cause des réticences du voisinage, quel que soit désormais le territoire dans lequel on réside. Cela freine considérablement le maintien et la diffusion de petits élevages. Même en territoire rural il reste peu de maisons isolées pour accueillir des mâles reproducteurs. Ce type d'élevage nécessite sur le long terme un investissement personnel et la mobilisation de terrains qui seront à perte en terme de rentabilité économique. Un éleveur doit compter sur dix ans de travail au moins avant de commencer à en récolter les fruits c'est-à-dire des sujets bien conformés.

Toutes ces raisons concourent à ce que le nombre d'exposants de races anciennes dans les expositions avicoles baisse de façon toujours plus importante et à ce que le nombre d'animaux exposés s'y réduise. Un exemple : la grande exposition avicole de Niort ne s'est pas tenue en 2018 faute d'un nombre suffisant de sujets à exposer alors qu'elle présentait, il y a encore 20 ans, quelque 3000 à 4000 cages. 


\section{Les effets induits de ces contraintes sur la survie des races anciennes}

La réduction préoccupante de nombreuses races anciennes de poules à des effectifs extrêmement bas induit des travers qui fragilisent un peu plus le maintien de ces races. La baisse des effectifs s'accélère même depuis dix ans environ en raison de nombreuses disparitions d'élevages qui les maintenaient encore.

Il faut 1000 sujets pour sauver une race. Certaines n'en comptent plus que quelques dizaines. À ce stade il faut opérer une retrempe pour espérer sauver la race. Pour éviter la consanguinité on effectue un croisement avec une race proche de celle à sauvegarder. On sélectionne alors au fils des générations dans les sujets issus de ce croisement, les spécimens les plus conformes au standard de la race que l'on veut sauver. Cependant la retrempe a un défaut majeur. Si l'on retrouve la forme, l'apparence d'une race avec cette technique, rien ne garantit que l'on retrouve les qualités gustatives de la race concernée. Le problème est similaire pour les races reconstituées c'est-à-dire des races disparues que des éleveurs ont reconstitué à partir de sélections issues de races proches d'elles. On réussit à reproduire des sujets conformes dans leur apparence au standard de la race à reconstituer mais aucune garantie n'est donnée sur la reconstitution des goûts des spécimens obtenus. Une perte irrémédiable s'est sûrement opérée. Les races reconstituées sont : la Caumont, la Crèvecoeur, la Cotentine, la poule de Janzé, la poule du Mans, la Mantes.

La dérive du travail de conservation est enfin un autre problème sérieux qui complique le maintien des races françaises de poules. Les éleveurs de ces races ont permis de les faire subsister jusqu'à aujourd'hui : grâce à eux, elles n'ont pas toutes disparues. Cependant il arrive désormais fréquemment que des éleveurs ne s'occupent dans la sélection des sujets d'une race que de leur conformité au standard de cette race ou ne s'occupent que de choisir des sujets en fonction d'un élément recherché par effet de mode et ce au détriment de la fécondité des spécimens retenus. Les éleveurs sélectionnent ainsi des sujets de moins en moins féconds ce qui accentue la baisse des effectifs de leur race.

Un exemple : il existe aujourd'hui des poules de Marans qui pondent très peu dans la mesure où leurs éleveurs ont seulement sélectionné des poules capables de pondre des œufs bien bruns, couleur très recherchée en ce moment, au détriment du nombre d'œufs qu'elles peuvent produire. En définitive la situation critique des trois-quarts des races de poules françaises - au bord de l'extinction - doit nous amener à considérer dans l'urgence comment établir des modèles d'élevage qui leur permettent de subsister et de se diffuser à nouveau dans l'ensemble des territoires, qu'ils soient urbains, périurbains ou ruraux. 
Une démarche de réflexion et de propositions autour de la sauvegarde des races de poules françaises

Face à cette situation de déclin des races françaises, Dominique Dubuc s'est engagé depuis une dizaine d'années dans une réflexion pour apporter sa contribution à la définition d'un modèle contemporain satisfaisant d'élevage de races de poules ${ }^{8}$. Dans cet esprit ce modèle doit mener à des produits de qualité (viande et oufs) c'est-à-dire des produits aux caractères gustatifs bien affirmés, qui présentent une palette riche de saveurs, odeurs, arômes, de sensations liées au goût suffisamment diversifiées. Ces informations sensorielles suggérant que les produits offrent des nutriments sains, bons pour la santé.

Ce processus de réflexion parait semblable, dans sa méthode, au processus qu'a connu le maraîchage en agroécologie : c'est-à-dire un réinvestissement de techniques anciennes éprouvées, notamment pour leur respect du vivant et pour la saveur des produits obtenus. Les techniques du maraîchage bio-intensif ont été remises à l'honneur à partir de leur redécouverte à la fin des années soixante par un mouvement qui a vulgarisé des techniques anciennes mises au point par les maraîchers français, techniques qui assuraient une production intensive de légumes savoureux et nourrissants tout en maintenant des sols écologiquement fertiles où leur biodiversité était maintenue. Ainsi que le rappelle Christian Carnavalet dans son ouvrage sur l'Agriculture biologique, deux Américains, Eliot Coleman et Alan Chadwick sont à l'origine de cette redécouverte de méthodes qu'ils ont puisées en particulier dans les écrits des jardiniers-maraîchers français Moreau et Daverne, publiés en 1845. En leur hommage, ils nomment Biodynamic French Intensive Method les techniques bio-intensives qu'ils diffusent et qui font peu à peu école auprès de maraîchers américains puis européens de plus en plus nombreux. Le maraîcher québécois bien connu, Jean-Martin Fortier, formateur en cultures bio-intensives, est un de leurs continuateurs.

Il a semblé que la poursuite de la réflexion de Dominique Dubuc sur les modèles d'élevage de races de poules suivait en ce sens une démarche similaire à celle de ces maraîchers réinvestissant des techniques anciennes éprouvées qui garantissaient des productions intensives et de haute qualité gustative et nutritionnelle sur des surfaces réduites. Il existe encore aujourd'hui des éleveurs de volailles pour continuer à faire vivre dans leur pratique quotidienne de l'aviculture des techniques anciennes éprouvées assurant des productions goûteuses et garantissant le maintien rigoureux des races anciennes dans leurs qualités et leur standard. Dominique Dubuc est de ceux-là. L'élevage qu'il pratique

8 À cet effet, en réponse à un concours organisé sur le thème de la sauvegarde des races anciennes par la Fondation du Patrimoine, il produit un mémoire en 2012 avec l'aide d'Hervé Goulaze : Projet de sauvegarde des races anciennes de volailles françaises à travers l'exemple de la poule landaise. 
perpétue des savoir-faire que l'on rencontrait encore dans l'Entre-deux-guerres - avant la massification et l'industrialisation de la production de volailles dans les centres de sélection des races les plus réputés comme l'était le Centre d'expérimentation zootechnique avicole de la ferme des Vaulx-de-Cernay, près de Paris. Il préconise de remobiliser et réemployer ces techniques qui étaient en honneur avant la Seconde Guerre mondiale. Elles étaient respectueuses de l'animal car les personnes qui les employaient pensaient que lorsque l'animal se sentait bien, on obtenait un produit de qualité qui se vendait bien. Ce respect de l'animal a disparu avec l'arrivée de la production de masse.

La mobilisation de l'expérience et du savoir paysan de Dominique Dubuc s'avère intéressante pour comprendre en quoi l'apport des techniques anciennes d'élevage avicole et des savoirs paysans qui y sont associés peut servir à la construction de nouvelles propositions d'élevage dans les territoires urbains, périurbains, ruraux où l'organisation spatiale et économique, où les filières et les paysages de production alimentaire ont été bouleversés en quelques décennies. Nous supposons que le recours à ces techniques peut aider à reconsidérer les paysages urbains, périurbains et ruraux à l'aune de leur capacité à accueillir un élevage offrant des produits intéressants par la diversité de leurs goûts comme par la qualité de leurs nutriments et à l'aune de contraintes générées par la structuration actuelle de ces territoires ainsi que par les conditions de vie qui leur sont associées. L'approche retenue évalue plusieurs esquisses de modèles d'élevage regardés sous l'angle de certaines contraintes incontournables et des nécessités d'un élevage tourné vers des produits goûteux, assurant des nutriments sains c'est-à-dire bons pour la santé.

\section{Le recours aux savoirs paysans dans l'élaboration de nouveaux modèles d'élevage : l'apport de Dominique Dubuc}

\section{Les nécessités et les principales contraintes retenues}

La cherté du foncier dans les territoires urbains et périurbains qui, avec la relative rareté des terrains utilisables pour de l'élevage, crée un manque de disponibilité de l'espace dont il convient de tenir compte en tout premier lieu.

Le respect de conditions d'élevage concourant à la valeur gustative et nutritionnelle des produits générés ainsi qu'au bien-être des sujets élevés. Seules des volailles bien traitées fourniront des produits goûteux.

La nécessité de la rentabilité de l'élevage dès lors qu'il est orienté vers des productions commerciales. Cet impératif est également maintenu lorsqu’il s'agit d'un élevage destiné à une production vivrière.

Le ressenti défavorable à l'égard des coqs et des nuisances sonores que beaucoup leur associent désormais, qui limite considérablement les possibilités d'installation de certains modèles d'élevage. 


\begin{tabular}{l|l|l|l|l|l|l}
$\begin{array}{l}\text { Orientation } \\
\text { de l'élevage }\end{array}$ & $\begin{array}{l}\text { Emprise au sol } \\
\text { des espaces } \\
\text { d'élevage }\end{array}$ & $\begin{array}{l}\text { Effectifs } \\
\text { (en nombre } \\
\text { de sujets) }\end{array}$ & $\begin{array}{l}\text { Contraintes } \\
\text { ou Exigences }\end{array}$ & $\begin{array}{l}\text { Activité } \\
\text { associée } \\
\text { recommandée }\end{array}$ & $\begin{array}{l}\text { Possibilité } \\
\text { d'élevage } \\
\text { urbain }\end{array}$ & $\begin{array}{l}\text { Possibilité } \\
\text { d'élevage } \\
\text { périurbain }\end{array}$ \\
\hline Reproduction & $\begin{array}{l}128 \mathrm{~m}^{2} \text { hors } \\
\text { poussinières }\end{array}$ & $\begin{array}{l}32 \\
\text { (8 coqs, } \\
24 \text { poules) }\end{array}$ & Chant du coq & $\begin{array}{l}\text { Difficile ou } \\
\text { Impossible } \\
\text { (en France) }\end{array}$ & $\begin{array}{l}\text { Difficile ou } \\
\text { Impossible } \\
\text { (en France) }\end{array}$ \\
\hline $\begin{array}{l}\text { Viande } \\
\text { pour } \\
\text { commercialisation }\end{array}$ & $\begin{array}{l}1000 \mathrm{~m}^{2} \text { sujets en } \\
\text { croissance } \\
220 \mathrm{~m}^{2} \text { sujets } \\
\text { adultes }\end{array}$ & 100 & $\begin{array}{l}\text { Surface d'élevage } \\
\text { Chant du coq }\end{array}$ & Impossible & $\begin{array}{l}\text { Possible mais } \\
\text { difficile }\end{array}$ \\
\hline $\begin{array}{l}\text { Oeufs et } \\
\begin{array}{l}\text { Poules grasses } \\
\text { (vieilles pondeuses) }\end{array}\end{array}$ & $\begin{array}{l}400 \mathrm{~m}^{2} \text { à la belle } \\
\text { saison } \\
105 \mathrm{~m}^{2} \text { en hiver }\end{array}$ & 100 & $\begin{array}{l}\text { Elevage en lien } \\
\text { avec l'arrière-pays } \\
\text { saisonnièrement }\end{array}$ & Maraîchage & $\begin{array}{l}\text { Possible } \\
\text { mais } \\
\text { difficile }\end{array}$ & Possible \\
\hline $\begin{array}{l}\text { Oeufs et } \\
\text { viande } \\
\text { production vivrière } \\
\text { pour une famille }\end{array}$ & $\begin{array}{l}44 \mathrm{~m}^{2} \text { sujets } \\
\text { adultes uniquement } \\
122 \mathrm{~m}^{2} \text { pour } 10 \\
\text { sujets juvéniles et } \\
10 \text { sujets adultes }\end{array}$ & 20 & $\begin{array}{l}\text { Elevage de poules } \\
\text { uniquement } \\
\text { pour éviter le chant du } \\
\text { coq }\end{array}$ & & Possible & Possible \\
\hline
\end{tabular}

Figure 3 : Tableau des modèles d'élevage esquissés

\section{Modèle d'élevage à des fins de reproduction}

Ce modèle destiné à reproduire les sujets d'une race comprend 8 volières de $16 \mathrm{~m}^{2}$ chacune, avec une emprise totale au sol de $128 \mathrm{~m}^{2}$. Chaque volière accueille 3 poules et un coq soit 32 sujets au total : 8 coqs et 24 poules. Les poussins sont élevés en poussinières. L'espace a été délibérément utilisé avec parcimonie. Il a cependant été déterminé avec soin pour correspondre aux besoins et au confort des volatiles qui y seront élevés. La réflexion initiale qui a abouti à cette proposition intègre déjà un souci d'économie de l'espace occupé, un trait qui la rend intéressante pour les territoires urbains et périurbains en particulier. Cependant cette proposition pose d'emblée le problème des coqs et de leur chant en milieu urbain et périurbain notamment. Ce problème paraît insoluble. Pour cette raison il est reste très difficile aujourd'hui encore d'envisager de réintroduire de l'élevage à des fins conservatoire en ville ou dans la périphérie des agglomérations. Les territoires ruraux eux-mêmes sont de plus en plus concernés par cette contrainte.

Ce problème, propre à la France, qui ne manque pas d'interpeller, résiste encore à l'explication. Les banlieues de certaines métropoles allemandes intègrent des terrains où des éleveurs se regroupent en club pour la reproduction de races de poules sans que cela pose problème.

Modèle de production de volailles de qualité pour la viande, à des fins commerciales

L'élevage des volailles de Bresse est pris pour référence dans ce modèle.

La surface nécessaire correspond à : 
- $10 \mathrm{~m}^{2}$ par juvénile soit $1000 \mathrm{~m}^{2}$ pour 100 sujets. Il s'agit d'une surface d'élevage spécifique aux sujets en croissance ;

- 2,2 $\mathrm{m}^{2}$ par sujet adulte, en plaçant 7 sujets dans une volière de $16 \mathrm{~m}^{2}$. Les sujets adultes ont besoin de moins d'espace que les juvéniles.

Ce modèle d'élevage ne peut se concevoir dans des territoires urbains du fait de la superficie qu'il exige pour l'élevage des juvéniles. Il est à réserver à des territoires ruraux ou périurbains occasionnellement, là où des terrains peuvent éventuellement rester disponibles et accessibles financièrement. De même la présence des coqs ne manquera pas de poser des problèmes de coexistence avec le voisinage.

\section{Modèle pour la production d'œufs à des fins commerciales}

Les effectifs nécessaires : une centaine de poules.

La superficie nécessaire :

- à la belle saison : 3,2 à $4 \mathrm{~m}^{2}$ par sujet, soit 320 à $400 \mathrm{~m}^{2}$ pour 100 sujets ;

- en hiver les poules sont en claustration : $0,75 \mathrm{~m}^{2}$. On pourra placer une quinzaine de poules dans un bâtiment de $15 \mathrm{~m}^{2}$. Soit environ 7 poulaillers de $15 \mathrm{~m}^{2}$ pour 100 sujets d'élevage. Les poules pondeuses sont en principe en claustration mais avec de l'éclairement et une nourriture appropriée.

Les œufs produits dans ce type d'élevage assurent un revenu complémentaire intéressant. Ce modèle est spécialement adapté à des maraîchers qui souhaiteraient compléter leur revenu par de la production d'œufs, des producteurs installés à la périphérie des villes. Certains maraîchers bio produisent déjà des œufs destinés à la vente.

Pour limiter le coût de la production, le maraîcher aurait intérêt à donner ses légumes abimés et ses invendus à ses volailles. Ce serait une manière de les valoriser et de renouer avec les pratiques des maraîchers d'autrefois qui associaient à leur activité principale, de l'élevage pour la production de viande et d'œufs à partir du recyclage des déchets de légumes. Ce modèle assure également une seconde production lucrative. Les poules prêtes à être reformées, les vieilles pondeuses grasses, sont recherchées pour faire de la poule-au-pot. Cette catégorie de volailles est devenue rare. On en tirera donc un bon prix quand elles auront cessé leur carrière de pondeuses.

L'élevage industriel a fait disparaître les poules grasses. Les industriels ont sélectionné des souches où le poids de la poule pondeuse a été réduit : elle ne mange que pour produire des œufs. On s'en débarrasse au bout d'un an. Elle servira à faire de la farine de viande ou de la nourriture pour chiens. C'est pourquoi le marché de la poule grasse est très intéressant aujourd'hui. Un des intérêts de ce modèle d'élevage viendrait de la reconstitution de réseaux de production liant les territoires situés dans la proximité de la ville avec l'arrière 
pays. C'est un souci créé par ce type d'élevage qui conduit à cette proposition car si l'espace laissé aux sujets est suffisant, il ne permet pas d'éviter le piquage. Les poules se piquent entre elles au moment du renouvellement de leurs plumes, se blessent et se causent beaucoup de stress, tout ceci contrariant beaucoup la production et le bien-être des volailles. L'enjeu serait d'arriver à ce que la production puisse se déplacer à certains moments de l'année, dans des lieux qui garantissent que les volailles aient suffisamment d'espace pour qu' il y ait moins de piquage. Cela suppose de mettre en relation des personnes et des territoires qui n'ont plus beaucoup l'habitude de travailler ensemble.

Pour ce faire il semble nécessaire de recréer des réseaux entre l'arrière pays et les territoires périurbains où seraient installés ces maraîchers de façon à ce qu'il passent des ententes avec leurs collègues de l'arrière pays, des agriculteurs prêts à leur louer de petits terrains où les maraîchers puissent mener leurs poules. Les maraîchers producteurs d'œufs installés dans des territoires périurbains pourraient ainsi avoir en permanence un roulement de sujets. Le modèle pour la production d'œufs est celui qui semble le plus adapté aux contraintes d'espaces disponibles dans les territoires urbains et périurbains. Quand on dispose de peu de place, la production d'oufs est la formule la plus raisonnable. De plus dans ce modèle où il n'y a pas de coq, il n'y a pas à envisager cette contrainte.

Cependant se limiter à n'avoir que des poules pour ne produire que des œufs et à terme des poules grasses signifie qu'il faut tout de même que s'effectue de la reproduction dans d'autres lieux pour renouveler les sujets adultes. Ainsi ce modèle nécessite que d'autres producteurs s'occupent de la reproduction pour fournir les poules pondeuses ou que le producteur d'œufs ait un terrain dans un autre lieu, propice à accueillir de la reproduction. Ce modèle implique en conséquence de mettre en relation des producteurs installés dans la ville ou à sa périphérie avec des producteurs installés dans l'arrière-pays, c'est-à-dire de constituer un réseau de producteurs implantés dans des territoires complémentaires du point de vue de la production et de l'économie de l'élevage.

Quelles sont les races adaptées à ce type d'élevage ? Il faut prendre des volailles qui ne soient pas farouches. La poule de Caussade avec son caractère très familier, convient bien.

La poule de Marans est très recherchée par les maraîchers bio producteurs d'œufs en raison de la couleur brun chocolat de sa coquille. Cependant cette race n'est pas une excellente pondeuse. Si on la choisit pour ce modèle d'élevage il sera préférable de travailler à partir d'un croisement avec une race qui soit meilleure pondeuse comme la Coucou de Rennes ou la New Hampshire. La sélection des meilleurs sujets pour la ponte permet d'améliorer sa souche en trois générations.

Si l'éleveur veut produire à partir d'une race pure, il prendra alors de la Coucou de Rennes qui est une bonne pondeuse. 


\section{Modèle pour la production de viande et œufs à des fins de consommation familiale}

Ce modèle nécessite les mêmes superficies par sujet que pour une production de viande à vocation commerciale soit $2,2 \mathrm{~m}^{2}$ par sujet adulte. Si l'on effectue soi-même l'élevage des juvéniles il faut $10 \mathrm{~m}^{2}$ par jeune sujet. Il faut cependant beaucoup moins de place que pour un élevage orienté vers la commercialisation. Dans le cas d'une production vivrière destinée à la famille les effectifs sont plus réduits. La superficie requise ne demande que $44 \mathrm{~m}^{2}$ pour 20 sujets adultes. Pour une famille de cinq personnes 10 poules d'un an et 10 poules de deux ans pourraient suffire. Tous les ans seraient intégrées des poulettes nées en avril/mai qui commenceraient à pondre à l'automne. À partir de ce moment on pourrait tuer les poules de deux ans et demie.

La démarche d'intégration d'un savoir paysan, de savoir-faire reposant sur des techniques anciennes d'élevage de volailles à la réflexion autour de la réintroduction d'un élevage proposant des produits goûteux s'appuyant sur les races anciennes de poules dans les territoires urbains, périurbains et ruraux constitue un recours fécond et pertinent pour travailler à la renaissance de paysages nourriciers proposant des produits intéressants d'un point de vue gustatif et nutritionnel, produits qui sont aux fondements de nouveaux terroirs.

L'approche initiale cherchait à considérer quel forme d'élevage à partir de races anciennes de poules pouvait s'accommoder des contraintes des environnements urbains et périurbains actuels tout en maintenant les exigences de la production pour aboutir à des produits goûteux qui proposent des nutriments sains, bons pour la santé.

La réflexion a été conduite vers des propositions inattendues. Elles paraissent montrer qu'il est également nécessaire de repenser les questions d'élevage et de production alimentaire de qualité en les abordant sous l'angle de la recréation de relations entre territoires, à une échelle plus vaste que celle envisagée originellement qui inclut l'arrière-pays, l'hinterland, avec la ville et ses paysages circonvoisins. Ces considérations ont pu apparaitre notamment parce que la réflexion a porté sur une tentative de conciliation de deux impératifs rarement confrontés : celui de réalités territoriales urbaines et périurbaines contraignantes pour l'élevage mais impératives et celui du respect rigoureux de critères d'élevage seuls à même de conduire à des productions offrant des qualités gustatives et nutritionnelles satisfaisantes.

\section{Dominique Dubuc}

Paysan, éleveur de volailles Créateur et responsable du Conservatoire agricole du Puyobrau 


\title{
Hervé Goulaze
}

\section{École Nationale Supérieure d'Architecture et de Paysage de Bordeaux herve.goulaze@bordeaux.archi.fr}

Hervé Goulaze est Paysagiste DPLG, diplômé de l'École Nationale supérieure d'architecture et de paysage de Bordeaux, doctorant en architecture et paysage (École Doctorale MontaigneHumanités et UMR Passages). Il est également enseignant à l'ENSAP de Bordeaux, créateur et coordonnateur du cours sur "La question alimentaire et les paysages ". Il s'intéresse aux possibilités de réintroduction de terroirs dans les territoires urbains et périurbains ainsi qu'au lien entre paysages nourriciers, terroir, caractéristiques gustatives et caractéristiques nutritionnelles des produits alimentaires.

\section{Résumé}

La majeure partie du patrimoine génétique, gustatif et nutritionnel constitué par les races anciennes de poules françaises menace de disparaittre. Les savoirs paysans sont un recours précieux pour concevoir de nouveaux modèles d'élevage de ces races. L'originalité de ces savoirs forgés par l'expérience de terrain tient notamment à ce qu'ils participent en permanence à la (re)création et à l'évolution de ce terrain. Ils sont à même de combiner à la fois les exigences économiques et spatiales fort contraignantes des territoires urbains et périurbains en matière d'élevage et les nécessités que demande un renouveau de l'élevage avicole s'appuyant sur les races anciennes, en particulier les caractéristiques techniques nécessaires à l'obtention de produits alimentaires sains, aux goûts affirmés et diversifiés. Ils aident à construire des modèles capables de faire renaître des élevages rentables au moindre coût, de taille modeste qui assurent la préservation des races considérées et du patrimoine qu'elles représentent. Recourir à ces savoirs paysans a également permis de mettre en évidence un impératif resté jusqu'alors sousjacent dans la renaissance d'un élevage de poules de races anciennes, pérenne et solidement installé d'un point de vue économique : la recréation indispensable de liens socio-économiques autour de cet élevage entre les territoires urbains, périurbains et les territoires ruraux.

\section{Mots-clés}

Volaille, poule, élevage, aviculture, race de volaille, race de poule, patrimoine avicole, élevage avicole, savoir paysan, terroir en formation, création de terroir, territoire urbain, territoire périurbain, territoire rural, élevage urbain, élevage périurbain.

\begin{abstract}
Most of the genetic, gustatory and nutritional heritage constituted by the ancient breeds of French hens is in danger of disappearing. Farmers' knowledge is a precious resource for designing new breeding models for these breeds. Developed by field experience, the originality of this knowledge, lies in the fact that it constantly participates in the (re)creation and evolution of this sector. We are able to combine both the highly restrictive economic and spatial requirements of urban and peri-urban territories in terms of breeding and the needs required by a renewal of poultry breeding based on old breeds, in particular the technical characteristics necessary to obtain healthy food products with strong and diversified tastes. The experience gained also helps to build models capable of reviving profitable, low-cost, small-scale poultry farms that preserve the breeds in question and the heritage they represent. Valorising the farmers' knowledge and skills in the revival of traditional chicken breeds, which is both economically viable and sustainable, has resulted in highlighting an antecedently forgotten necessity of the indispensable re-creation of socio-economic links between urban, peri-urban and rural areas.
\end{abstract}

\section{Keywords}

Poultry, hen, breeding, poultry farming, poultry breed, chicken breed, poultry heritage, poultry farming, farming knowledge, terroir in the process of being formed, creation of terroir, urban territory, peri-urban territory, rural territory, urban breeding, peri-urban breeding. 